\title{
A MIXTURE THEOREM FOR NONCONSERVATIVE MECHANICAL SYSTEMS*
}

BY A. H. COPELAND $\dagger$

We shall be concerned with a mechanical system which is acted upon by friction in such a manner that it eventually comes to rest. We shall assume that there is a probability distribution for the initial conditions of the system. There is then determined a corresponding distribution for the rest position. We shall show that in general as the friction approaches zero, this latter distribution approaches uniformity irrespective of the initial distribution. This type of problem was first solved by Poincaréf The theory was later developed by Smoluchowski§ and recently an extensive contribution based on the ergodic theorem $\|$ has been made by Hopf. $\uparrow$

We shall let $x_{1}, x_{2}, \cdots, x_{\nu}$ denote the position coordinates of the system at any time $t$ and shall let $x$ denote the vector $x_{1}, x_{2}, \cdots, x_{\nu}$. Thus $d x / d t$ is the velocity vector. The initial position will be denoted by the vector $B$ and the initial velocity by the vector $V$. Then $V, B$ can be any point of $2 \nu$-dimensional space $\Omega$. We assume that there exists a continuous function $\psi$ such that the probability of the initial conditions being represented by a point $V, B$ in a given region $S$ is equal to the integral of $\psi$ taken over $S$. This condition is expressed by the equation

$$
\operatorname{prob}[(V, B) \epsilon S]=\int_{S} \psi(V, B) d \omega
$$

\footnotetext{
* Presented to the Society, December 27, 1934.

$\dagger$ Guggenheim fellow.

$\ddagger$ Calcul des Probabilités, 1912.

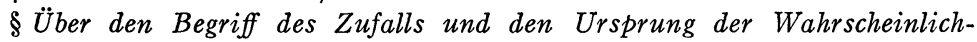
keitsgesetze in der Physik, Die Naturwissenschaften, vol. 6 (1918).

\| Birkhoff, Proof of a recurrence theorem for strongly transitive systems, Proof of the ergodic theorem, Proceedings of the National Academy of Sciences, vol. 17 (1931).

I On causality, statistics, and probability, Journal of Mathematics and Physics, Massachusetts Institute of Technology, vol. 13 (1934). See also Struik, On the foundations of the theory of probability, Philosophy of Science, vol. 1 (1934).
} 
Since $\psi$ is a distribution function, it must satisfy the relation

$$
\int_{\Omega} \psi(V, B) d \omega=1 .
$$

Otherwise $\psi$ will be unrestricted.

We shall assume that the coordinates $a_{1}, a_{2}, \cdots, a_{\nu}$ of the rest position are angular variables. That is, we shall be interested in the values of these variables reduced by some modulus. By a proper choice of units, we can make this modulus equal to 1. Thus we shall study the probability distribution for the vector $a-[a]$ whose components are $a_{1}-\left[a_{1}\right], a_{2}-\left[a_{2}\right], \cdots, a_{\nu}-\left[a_{\nu}\right]$, where $\left[a_{i}\right]$ is the largest integer contained in $a_{i}$. The vector $a-[a]$ lies in the region $\Delta$ given by the inequalities $0 \leqq z_{i}<1$, where $i=1,2, \cdots, \nu$. The probability distribution of $a-[a]$ will be called uniform if for every region $E$ of $\Delta$, we have the equation

$$
\operatorname{prob}(a-[a] \epsilon E)=m(E),
$$

where $m(E)$ is the $\nu$-dimensional Lebesgue measure of $E$. The region $E$ may be any set whose frontier points constitute a set of measure 0 . Our problem is to show that this uniform distribution is approached as the friction is decreased.

We shall first consider a system defined by the equation

$$
\frac{d^{2} x}{d t^{2}}=\mu \cdot f\left(\frac{d x}{d t}\right),
$$

where $\mu$ is a scalar parameter. If we substitute $x=B+\xi / \mu$ and $t=\tau / \mu$, then we observe that $d x / d t=d \xi / d \tau$ and $d^{2} x / d t^{2}=\mu d^{2} \xi / d \tau^{2}$. Hence

$$
\frac{d^{2} \xi}{d \tau^{2}}=f\left(\frac{d \xi}{d \tau}\right) .
$$

We can obtain a solution $\xi=X(V, \tau), d \xi / d \tau=\dot{X}(V, \tau)$ such that $X(V, 0)=0$ and $\dot{X}(V, 0)=V$. After a time $T$, the system (5) comes to rest. Thus $X(V, T)=A$ and $\dot{X}(V, T)=0$, where $A$ is the rest position vector. If we solve one of the equations $\dot{X}(V, T)=0$ for $T$ in terms of $V$ and substitute this value in the equations $X(V, T)=A$, we obtain the relations $A=F(V)$ 
and $V=F^{-1}(A)$. We shall assume that system (4) (or (5)) is such that the Jacobian $J\left(\begin{array}{l}V \\ A\end{array}\right)$ is continuous and non-vanishing. Then

$$
\int_{S} \psi(V, B) d \omega=\int_{R} \phi(A, B) d \sigma=\operatorname{prob}[(A, B) \epsilon R],
$$

where $\phi(A, B)=\psi\left[F^{-1}(A), B\right] \cdot J\left(\begin{array}{l}V \\ A\end{array}\right)$ and $R$ is the transformed region $S$.

It will be observed that $x=B+X(V, \mu t) / \mu, d x / d t=\dot{X}(V, \mu t)$ is the solution of system (4) whose initial conditions are represented by the point $V, B$ of the phase space $\Omega$. The rest position of this system is the vector $a=B+A / \mu$. In order to study the distribution of $a-[a]$, we shall introduce a vector $b=-B / \mu+A$ and two systems of regions

$$
\begin{aligned}
& r_{m, n}: \quad m+\alpha \leqq a<m+\beta, \quad n \leqq b<n+1, \\
& R_{m, n}: \quad m \leqq a<m+1, \quad n \leqq b<n+1 \text {, }
\end{aligned}
$$

where $m$ and $n$ stand respectively for the sets of integers $m_{1}, m_{2}, \cdots, m_{\nu}$ and $n_{1}, n_{2}, \cdots, n_{\nu}$. Then the set of all points $A, B$ for which $\alpha \leqq a-[a]<\beta$ is $\sum_{m, n} r_{m, n}$, where the summation is extended over integral values of $m_{1}, m_{2}, \cdots, m_{\nu}, n_{1}, n_{2}, \cdots, n_{\nu}$. Hence

$$
\begin{aligned}
\operatorname{prob}(\alpha \leqq a-[a]<\beta) & =\sum_{m, n} \operatorname{prob}\left[(A, B) \epsilon r_{m, n}\right] \\
& =\sum_{m, n} \int_{r_{m, n}} \phi(A, B) d \sigma .
\end{aligned}
$$

The set of all points $A, B$ is the region $\Gamma=\sum_{m, n} R_{m, n}$.

We shall apply the mean value theorem to evaluate the integrals occurring in this latter summation. To accomplish this, it will be necessary to compute the volumes of the regions $r_{m, n}$. An equation $b_{i}=-B_{i} / \mu+A_{i}$, where $b_{i}$ is a constant, represents a line in the $A_{i} B_{i}$ plane. If $\theta$ is the angle between such a line and the positive $A_{i}$ axis, then $\tan \theta=\mu$. In a similar manner, an equation $a_{i}=B_{i}+A_{i} / \mu$, where $a_{i}$ is a constant, is a line with slope $-1 / \mu$. Hence the two inequalities $m_{i}+\alpha_{i} \leqq a_{i}<m_{i}+\beta_{i}$, $n_{i} \leqq b_{i}<n_{i}+1$, determine a rectangle whose area is

$$
\left(\beta_{i}-\alpha_{i}\right) \cdot \sin ^{2} \theta=\left(\beta_{i}-\alpha_{i}\right)\left(\frac{\mu^{2}}{1+\mu^{2}}\right) .
$$


It follows that the volume of $r_{m, n}$ is

$$
\prod_{i=1}^{\nu}\left(\beta_{i}-\alpha_{i}\right) \cdot\left(\frac{\mu^{2}}{1+\mu^{2}}\right)^{\nu} .
$$

In a similar manner it can be seen that the volume of $R_{m, n}$ is $\left[\mu^{2} /\left(1+\mu^{2}\right)\right]^{\nu}$. It follows from the mean value theorem that

$$
\begin{aligned}
\operatorname{prob}(\alpha \leqq a & -[a]<\beta) \\
& =\sum_{m, n} \phi\left(\bar{A}_{m, n}, \bar{B}_{m, n}\right) \prod_{i=1}^{\nu}\left(\beta_{i}-\alpha_{i}\right)\left(\frac{\mu^{2}}{1+\mu^{2}}\right)^{\nu} \\
& =\prod_{i=1}^{\nu}\left(\beta_{i}-\alpha_{i}\right) \sum_{m, n} \phi\left(\bar{A}_{m, n}, \bar{B}_{m, n}\right)\left(\frac{\mu^{2}}{1+\mu^{2}}\right)^{\nu},
\end{aligned}
$$

where $\bar{A}_{m, n}, \bar{B}_{m, n}$ is a properly chosen point of the region $r_{m, n}$. Since $\bar{A}_{m, n}, \bar{B}_{m, n}$ is also a point of $R_{m, n}$ and since $\left[\mu^{2} /\left(1+\mu^{2}\right)\right]^{\nu}$ is the volume of this region, it follows from the definition of the definite integral that

$$
\begin{aligned}
\lim _{\mu \rightarrow 0} \operatorname{prob}(\alpha \leqq a-[a]<\beta) & =\prod_{i=1}^{\nu}\left(\beta_{i}-\alpha_{i}\right) \int_{\Gamma} \phi(A, B) d \sigma \\
& =\prod_{i=1}^{\nu}\left(\beta_{i}-\alpha_{i}\right) .
\end{aligned}
$$

Thus we have proved that

$$
\lim _{\mu \rightarrow 0} \operatorname{prob}\left(a-[a]_{\epsilon E}\right)=m(E),
$$

if $E$ is the rectangular domain $\alpha \leqq z<\beta$. It remains to show that equation (11) holds for any set $E$ whose frontier points are of measure 0 . Corresponding to any such set and to any given positive number $\epsilon$, there exist two sets $E_{1}$ and $E_{2}$ such that each consists of a finite sum of rectangular regions, that $E_{1}<E<E_{2}$, and that $m\left(E_{1}-E_{2}\right)<\epsilon{ }^{*}$ Hence it is easily seen that equation (11) holds for such sets.

Next we shall consider any mechanical system defined by second order differential equations involving a parameter $\mu$.

* For a proof of this statement, see the author's article Admissible numbers in the theory of geometrical probability, American Journal of Mathematics, vol. 53 (1931). 
The solution of these equations has the form $x=X(V, B, t, \mu)$, $d x / d t=\dot{X}(V, B, t, \mu)$, where $X(V, B, 0, \mu)=B$ and $\dot{X}(V, B, 0, \mu)$ $=V$. We shall suppose that after a time $T$ the system comes to rest. Thus $X(V, B, T, \mu)=a$ and $\dot{X}(V, B, T, \mu)=0$. Solving one of the equations $\dot{X}(V, B, T, \mu)=0$ for $T$ and substituting this value in the function $X(V, B, t, \mu)$, we obtain the relations $a=F(V, B, \mu)$ and $V=F^{-1}(a, B, \mu)$. We shall suppose that the differential system is such that the limit as $\mu$ approaches 0 of $\mu \cdot F(V, B, \mu)$ exists and is not equal to 0 . It is then natural to make the substitution $u=a \mu=\mu F(V, B, \mu)=f(V, B, \mu)$. We shall let $A=F(V, B, 1)$ and hence $V=F^{-1}(A, B, 1)$. Substituting this value of $V$ in the function $F(V, B, \mu)$, we obtain the relations $a=G(A, B, \mu)$ and $u=g(A, B, \mu)$. We shall define a second system of equations $v=h(A, B, \mu)$ and shall assume that the functions $h$ can be so determined that the partial derivatives $\partial A_{i} / \partial u_{j}, \partial A_{i} / \partial v_{j}, \partial B_{i} / \partial u_{j}, \partial B_{i} / \partial v_{j}$ are bounded and uniformly continuous throughout the range of the points $u, v$ and for all values of $\mu$ in the interval $0 \leqq \mu \leqq 1$. Then $J\left(\begin{array}{ll}A, & B \\ u, & v\end{array}\right)$ is bounded and uniformly continuous. We shall assume also that this Jacobian is non-vanishing.

We shall again define two systems of regions

$$
\begin{aligned}
r_{m, n}: & \mu(m+\alpha) & \leqq u<\mu(m+\beta), & & \mu n \leqq v<\mu(n+1), \\
R_{m, n}: & \mu m & \leqq u<\mu(m+1), & & \mu n \leqq v<\mu(n+1) .
\end{aligned}
$$

The diameters of $R_{m, n}$ and $r_{m, n}$ (considered as regions of the space $\Gamma$ ) approach 0 with $\mu$. We have the equation

$$
\begin{aligned}
\operatorname{prob}(\alpha & \leqq a-[a]<\beta)=\sum_{m, n} \int_{r_{m, n}} \phi(A, B) d \sigma \\
= & \sum_{m, n} \phi\left(\bar{A}_{m, n}, \bar{B}_{m, n}\right) \cdot J\left(\begin{array}{cc}
A, & B \\
\bar{u}_{m, n}, \bar{v}_{m, n}
\end{array}\right) \prod_{i=1}^{\nu}\left(\beta_{i}-\alpha_{i}\right) \mu^{-2 \nu},
\end{aligned}
$$

where $\phi(A, B)=\psi\left[F^{-1}(A, B, 1), B\right] \cdot J\left(\begin{array}{l}V \\ A\end{array}\right)$ and $\bar{u}_{m, n}, \bar{v}_{m, n}$ is a properly chosen point of the region $r_{m, n}$. Equation (13) can also be written in the form

$$
\begin{aligned}
& \text { prob }(\alpha \leqq a-[a]<\beta) \\
& =\prod_{i=1}^{\nu}\left(\beta_{i}-\alpha_{i}\right) \sum_{m, n} \phi\left(\bar{A}_{m, n}, \bar{B}_{m, n}\right) J\left(\begin{array}{cc}
A, & B \\
\bar{u}_{m, n}^{\prime}, & \bar{v}_{m, n}^{\prime}
\end{array}\right) \mu^{-2 \nu}\left(1+\epsilon_{m, n}\right),
\end{aligned}
$$


where

$$
J\left(\begin{array}{cc}
A, & B \\
\bar{u}_{m, n}^{\prime}, & \bar{v}_{m, n}^{\prime}
\end{array}\right) \mu^{-2 v}
$$

is the volume of $R_{m, n}$ and $\epsilon_{m, n}$ approaches 0 uniformly with $\mu$. Therefore

It follows that

$$
\begin{aligned}
\lim _{\mu \rightarrow 0} \operatorname{prob}(\alpha \leqq a-[a]<\beta) & =\prod_{i=1}^{\nu}\left(\beta_{i}-\alpha_{i}\right) \int_{\Gamma} \phi(A, B) d \sigma \\
& =\prod_{i=1}^{\nu}\left(\beta_{i}-\alpha_{i}\right) .
\end{aligned}
$$

$$
\lim _{\mu \rightarrow 0} \operatorname{prob}\left(a-[a]_{\epsilon E)}=m(E)\right.
$$

for every set $E$ whose frontier points are of measure 0 . Thus the distribution of $a-[a]$ approaches uniformity as the parameter $\mu$ approaches 0 .

We shall consider two simple extensions of this theory. We may be concerned with problems involving only $\lambda$ of the coordinates of the rest position where $\lambda<\nu$. In this case it is only necessary to observe that any sub-set of the sub-space of $\lambda$ dimensions is also a sub-set of the space $\Delta$ and that if the frontier points of such a set are of measure 0 with respect to the subspace, they are also of measure 0 with respect to $\Delta$. Hence a uniform distribution is approached in the sub-space.

Suppose that we make a series of $n$ experiments with a mechanical system and that the initial conditions for these experiments are dependent. We shall inquire the limiting value of the probability that the rest positions of the 1 st, 2 nd, $\cdots, n$th experiments will fall respectively in the sets $E_{1}, E_{2}, \cdots, E_{n}$. This problem is essentially the same as those previously considered. Thus we have a system of $n \nu$ second order differential equations obtained by repeating the $\nu$ equations $n$ times. We have a corresponding distribution function for the initial conditions which is defined throughout a phase space of $2 n \nu$ dimensions. It follows that the probability in question is $m\left(E_{1}\right) \cdot m\left(E_{2}\right) \cdots m\left(E_{n}\right)$. Hence in the limit we have independence with respect to the rest positions even though we had dependence in the initial conditions.

The University of MichigaN 